\title{
Trends in COPD prevalence and exacerbation rates in Dutch primary care
}

\author{
Erik WMA Bischoff, Tjard RJ Schermer, Hans Bor, Pete Brown,
} Chris van Weel, Wil JHM van den Bosch

\author{
ABSTRACT \\ Background \\ Changes in the burden of chronic obstructive \\ pulmonary disease (COPD) and its exacerbations on \\ primary health care are not well studied. \\ Aim \\ To identify trends in the prevalence of physician- \\ diagnosed COPD and exacerbation rates by age, sex, \\ and socioeconomic status in a general practice \\ population. \\ Design of study \\ Trend analysis of COPD data from a 27-year \\ prospective cohort of a dynamic general practice \\ population. \\ Setting \\ Data were taken from the Continuous Morbidity \\ Registration Nijmegen. \\ Method \\ For the period 1980-2006, COPD and COPD \\ exacerbation data were extracted for patients aged \\ $\geq 40$ years. Data were standardised for the composition \\ of the Continuous Morbidity Registration population in \\ the year 2000. Regression coefficients for trends were \\ estimated by sex, age, and socioeconomic status. \\ Rate ratios were calculated for prevalence differences \\ in different demographic subgroups. \\ Results \\ During the study period, the overall COPD prevalence \\ decreased from 72.7 to 54.5 per 1000 patients per \\ year. The exacerbation rate decreased from 44.1 to \\ 31.5 per 100 patients, and the percentage of patients \\ with COPD who had exacerbations declined from \\ $27.6 \%$ to $21.0 \%$. The prevalence of COPD increased \\ significantly in women, in particular those aged \\ $\geq 65$ years with low socioeconomic status. Decreases in \\ exacerbation rates and percentages of patients with \\ exacerbations were independent of sex, age, and \\ socioeconomic status. \\ Conclusion \\ The decline in COPD prevalence and exacerbation \\ rates suggests a reduction of the burden on Dutch \\ primary care. The increase of the prevalence in women \\ indicates a need to focus on this particular subgroup in \\ COPD management and research. \\ Keywords \\ chronic obstructive pulmonary disease; family practice; \\ prevalence; trends.
}

\section{INTRODUCTION}

The prevalence of chronic obstructive pulmonary disease (COPD) and its subsequent burden on health care differ from country to country but is extensive in most populations. ${ }^{1}$ COPD exacerbations contribute substantially to the burden by generating the majority of costs due to the use of healthcare services ${ }^{2}$ and by provoking significant impact on patients' health status. ${ }^{3}$ In the Netherlands, the burden of COPD mainly affects GPs as most patients with COPD are managed in primary care; as such, there is a growing need to identify changes in the burden and to redirect care to those demographic subgroups that are at highest risk of COPD and its exacerbations. In this context, trend studies may be very useful. So far, most COPD trend studies have used data from national health surveys to estimate trends in the general population, ${ }^{4,5}$ and so their results may not reflect the burden in general practice.

A recent trend study using data from a general

EWMA Bischoff, $M S c, M D, G P$, junior researcher;

TRJ Schermer, PhD, epidemiologist, senior researcher; $\boldsymbol{H}$ Bor, $B S c$, statistician; C van Weel, PhD, MD, FRCGP, GP and professor in general practice; WJHM van den Bosch, $P h D$, $M D, G P$ and professor in general practice, Department of Primary and Community Care, Radboud University Nijmegen Medical Centre, Nijmegen, The Netherlands. P Brown, medical student, Imperial College, London, UK.

Address for correspondence

Erik WMA Bischoff, Department of Primary and Community Care, HAG 117, Radboud University Nijmegen Medical Centre, PO Box 9501, 6500 HB Nijmegen, The Netherlands. E-mail: e.bischoff@hag.umcn.nl

Submitted: 7 January 2009; Editor's response: 20 February 2009; final acceptance: 29 April 2009.

(c)British Journal of General Practice.

This article was originally online first on 4 Nov 2009. Cite this article as: Br J Gen Pract 2009; 59: 927-933. Advance online publication. DOI: 10.3399/bjgp09X473079. 


\section{How this fits in}

Changes in the burden of chronic obstructive pulmonary disease (COPD) on primary care are not well studied. This long-term trend study demonstrated a decrease in the overall prevalence of physician-diagnosed COPD and in exacerbation rates in Dutch general practices. However, the prevalence of COPD has increased in women, in particular those aged $\geq 65$ years with low socioeconomic status. These results indicate the need to focus more on this subgroup in COPD diagnosing, management and research.

practice network demonstrated a constant rise of physician-diagnosed COPD in women. ${ }^{6}$ This could be caused by the increase of tobacco use in women relative to men in the past few decades. The risk of developing COPD in smokers is related to the lifetime cumulative dose, which explains the finding that it is mostly diagnosed in people aged $\geq 65$ years and is highly unlikely in those aged $<40$ years. ${ }^{5}$ Independent from smoking, socioeconomic status is an important risk factor for COPD. ${ }^{7}$ In addition, factors linked to socioeconomic status, such as respiratory tract infections, housing conditions, air pollution, and diet each contribute to the risk of having exacerbations..$^{8-10}$ Obviously, sex, age, and socioeconomic differences are important factors that can influence trend changes of COPD and exacerbations. Although these factors are well registered in general practice registration networks, their roles have not been well studied in COPD trend studies in primary care.

In this article, long-term trends in physiciandiagnosed COPD prevalence and COPD exacerbations in the period 1980-2006 are reported by prospectively studying a dynamic cohort from the Continuous Morbidity Registration Nijmegen. The aim was to identify trend changes in COPD prevalences and COPD exacerbation occurrence rates in different subgroups of the general practice population.

\section{METHOD \\ Setting}

The Continuous Morbidity Registration of the Department of Primary and Community Care of the Radboud University Nijmegen Medical Centre is a prospective cohort study in which four Dutch general practices with a registered practice population of approximately 15000 subjects in the Nijmegen area have participated since 1967. It is a relatively stable practice population that reflects the Dutch healthcare system, that is, all patients are registered with a GP and all access to care is through referral by the GP. ${ }^{11}$ Since its founding, the Continuous Morbidity Registration Nijmegen has served as a successful practice-based research network for many primary care studies. ${ }^{12}$

The GPs of the Continuous Morbidity Registration practices record all diagnoses of all new episodes of illness in a standardised way, based on the E-list that is used to register morbidities in general practice ${ }^{13}$ and the International Classification of Health Problems in Primary Care. ${ }^{14}$ Recurrence of an episode after successful treatment or natural recovery (that is, a patient reporting that respiratory symptom aggravation recurred after a period in which exacerbation symptoms had returned to baseline level) is considered as a new episode. Chronic conditions such as COPD are coded annually after the year of initial diagnoses. ${ }^{15}$ Diagnoses made by specialists after referral are reported back to the GP and are entered in the database. The validity of the recorded diagnoses in the Continuous Morbidity Registration has been shown to be well above $80 \%$ in a variety of conditions. ${ }^{16,17}$

\section{Subjects and definition of variables}

Patients diagnosed with COPD were identified for the period 1980-2006 and were included in the annual prevalence rates from the first year of diagnosis. All patients were retrieved from the database with the E-list codes 'chronic bronchitis', 'lung emphysema', and 'COPD'. In the Continuous Morbidity Registration, COPD exacerbation was defined as an acute episode of respiratory symptom worsening, for which the patient, registered with a diagnostic code compatible with COPD, consulted the GP. Diagnosed exacerbations were recorded separately by adding a unique code for exacerbations to the diagnostic code for COPD or by using the E-list code 'acute bronchitis' or 'chronic bronchitis' in patients previously diagnosed with COPD. Exacerbation follow-up contacts were not considered as new events.

A small and, in time, significantly decreasing proportion of patients aged $<40$ years was observed in the Continuous Morbidity Registration. Given this small proportion and the opinion that COPD is highly unlikely in subjects aged $<40$ years, trend analyses were limited to data from patients with COPD who were aged $\geq 40$ years. Age was grouped into two bands: $40-64$ years and $\geq 65$ years. Socioeconomic status was determined by classifying the occupation of the patient using the Netherlands Standard Classification of Occupations 1992. ${ }^{18}$ The coded occupations were ranked into low, medium, and high socioeconomic status. Due to very low prevalence rates and small group sizes in the high socioeconomic status group, the medium and high socioeconomic status groups were joined in the 


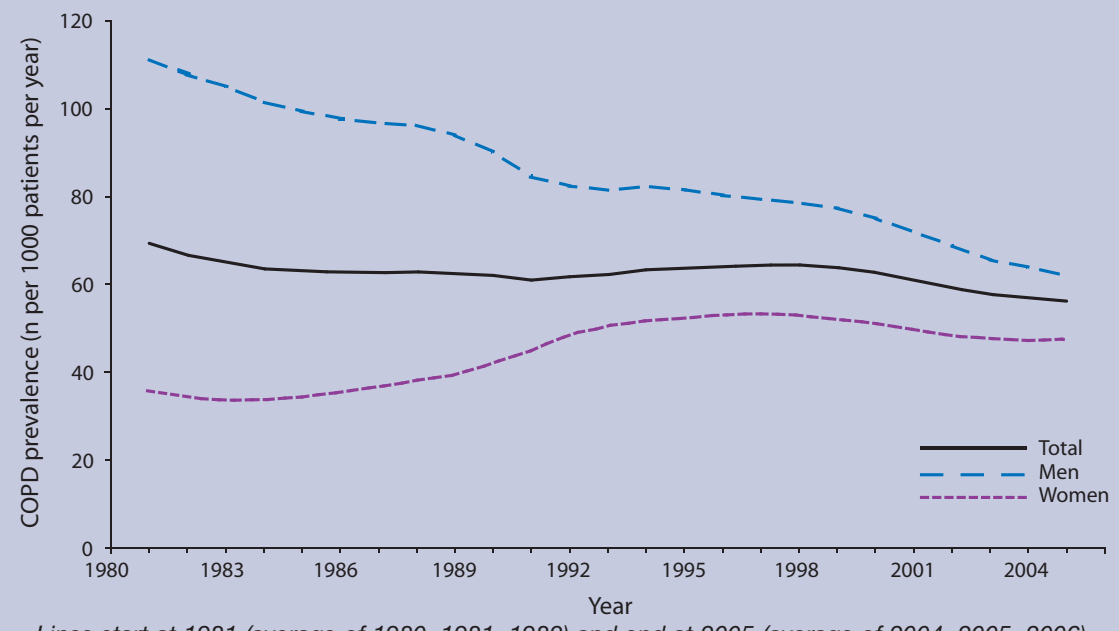

Figure 1. Trends in prevalence (n per 1000 patients per year), of physician-diagnosed COPD by sex; 3-year moving averages for the period 1980 to 2006.

trend analyses and will be presented as 'medium to high socioeconomic status'.

\section{Statistical analyses}

The number of patients with COPD and the frequency of exacerbations per patient were counted annually. Prevalence and exacerbation rates were standardised for the composition of the Continuous Morbidity Registration population in the year 2000 by age, sex, and socioeconomic status. Univariate regression analyses were conducted on the annual COPD prevalences and exacerbation rates using SPSS (version 16.0.2., SPSS Inc, Chicago). Regression coefficients for trends (RC trends) should be interpreted as the annual change in prevalence per 1000 patients per year. An RC trend was considered statistically significant if the $95 \%$ confidence interval (Cl) did not include 0 and if $P<0.05$.
Rate ratios (RRs) were calculated by dividing the annual prevalence or occurrence rate of two different subgroups (that is, males versus females, medium to high socioeconomic status versus low socioeconomic status, 40-64 years versus $\geq 65$ years).

In the exacerbation data an apparent split was observed in 1986 that may have been caused by a change in the registration of exacerbations; therefore, regression analyses were conducted on exacerbation data from 1986 onwards. Figures 2 and 3 presenting exacerbation rates also show data from 1980 to 1985.

Prevalence rates are presented as the number of COPD cases per 1000 patients per year and exacerbation rates as the number of exacerbations per 100 patients with COPD per year..$^{15}$ The presented Figures 1-3 show 3-year moving average

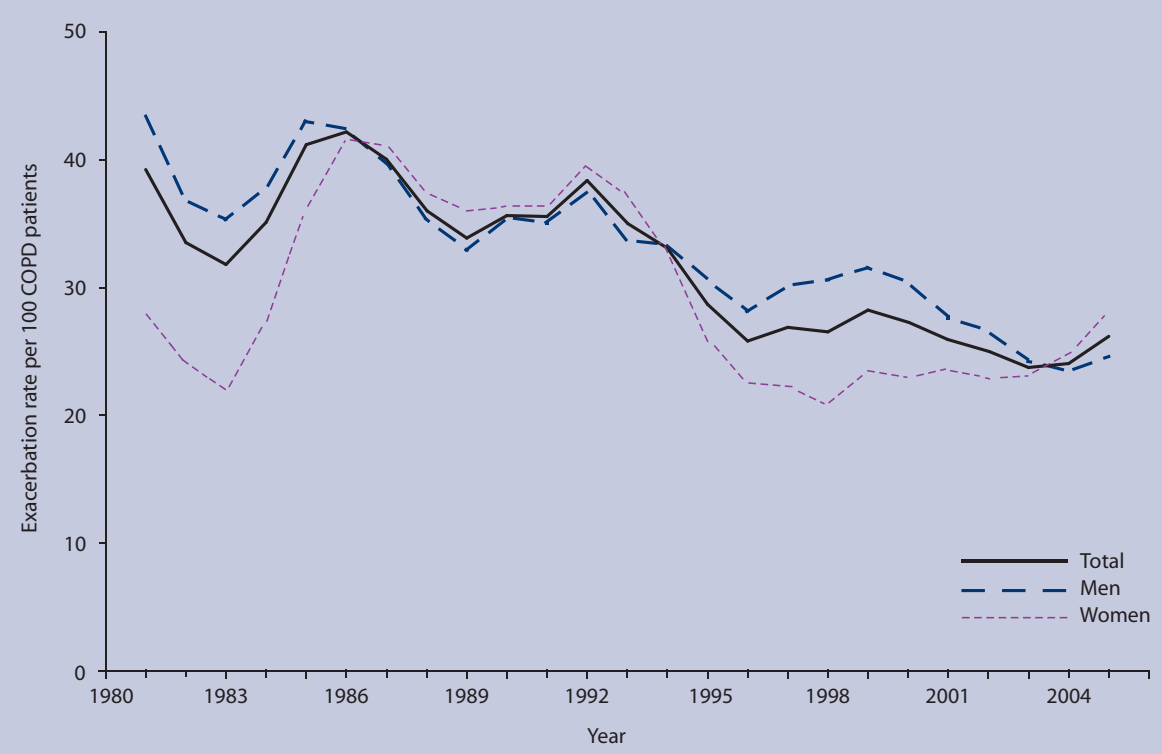

Lines start at 1981 (average of 1980, 1981, 1982) and end at 2005 (average of 2004, 2005, 2006).

Figure 2. Trends in exacerbation rates (n per 100 patients per year) of patients with physiciandiagnosed COPD; 3-year moving averages for the period 1980 to 2006 Trends were analysed for the period 1986-2006. 


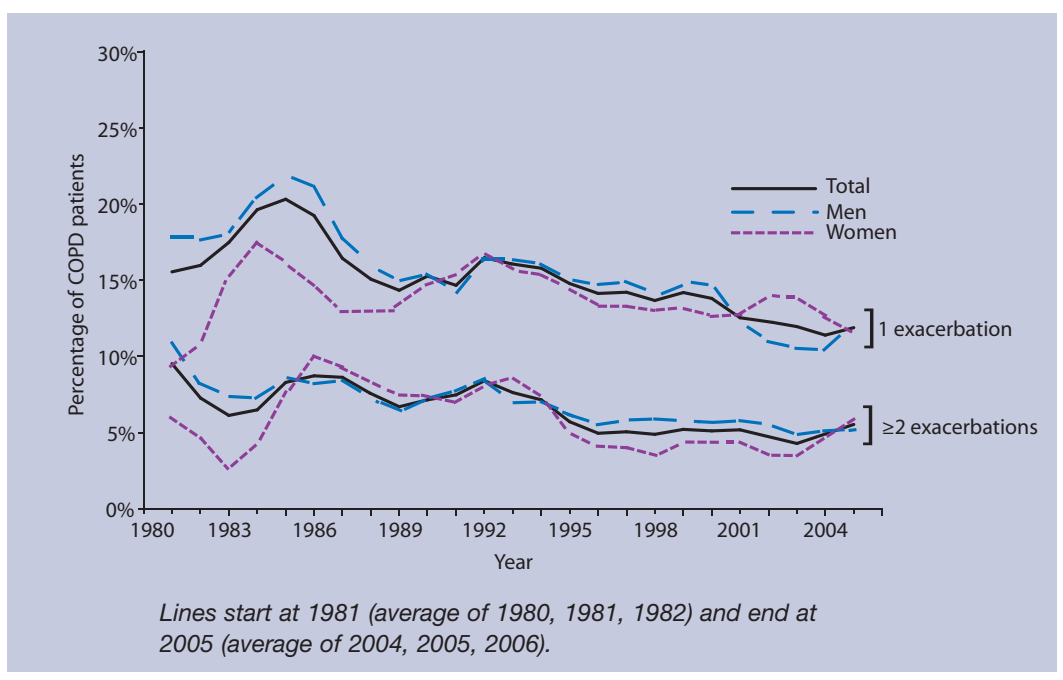

Figure 3. Trends in COPD patients with exacerbations (\% of total COPD patients): 3-year moving averages for the period 1980-2006. Trends were analysed for the period 1986-2006. rates to reduce the effect of random variation in consecutive calendar years.

\section{RESULTS \\ Trends in COPD}

In 1980 , a total of 423 patients aged $\geq 40$ years with physician-diagnosed COPD could be detected in the Continuous Morbidity Registration population. The prevalence per 1000 patients per year was 72.7 . In 2006, the number of patients with COPD had decreased to 317 with a prevalence of 54.4 patients per 1000 patients per year. Figure 1 shows trends in COPD prevalence for the total cohort and for men and women separately. In 1980, COPD prevalence in men was higher than in women $(R R=3.1)$, but decreased from 115.2 patients per 1000 patients per year to 59.0 patients per 1000 patients per year (RC trend $=-1.92,95 \% \mathrm{Cl}=-2.06$ to -1.77 ) in 2006. In contrast, the COPD prevalence in women increased from 37.5 patients per 1000 patients per year in 1980 to 47.2 patients per 1000 patients per year in $2006(\mathrm{RC}$ trend $=0.73,95 \% \mathrm{Cl}=0.49$ to 0.96$)$, thereby closing the gender gap $(\mathrm{RR}=1.2)$. Table 1 presents prevalence estimates by sex combined with age and socioeconomic status.

In men and women the prevalence rates were highest in patients aged $\geq 65$ years with a low socioeconomic status. The strongest increase in COPD prevalence was observed in women of low socioeconomic status aged $\geq 65$ years (prevalence difference 1980-2006 $=46.5$ per 1000 patients per year, $\mathrm{RC}$ trend $=2.55,95 \% \mathrm{Cl}=2.02$ to 3.09 ), while the strongest decrease in COPD prevalence was demonstrated in men of low socioeconomic status aged $\geq 65$ years (prevalence difference 1980-2006 = -134.1 per 1000 patients per year, RC trend $=-5.17$, $95 \% \mathrm{Cl}=-6.23$ to -4.12 ). In all male subgroups the COPD prevalence trends showed a statistically significant decrease in the regression analyses.

\section{Trends in COPD exacerbations}

From 1980 until 2006, a total of 2831 exacerbations were recorded by the GPs in the Continuous Morbidity Registration practices. Figure 2 presents trend curves for the annual exacerbation rates in the total COPD population and in men and women separately. The trend curve in the total COPD cohort showed a gradual decline from 1986 onwards, from 45.2 to 31.5 exacerbations per 100 patients with COPD. The estimated trend regression coefficient showed a decrease of 0.82 exacerbations per 100 COPD patients per year (95\% $\mathrm{Cl}=-1.14$ to -0.50$)$. This trend was also observed in men $(\mathrm{RC}$ trend $=-0.77,95 \% \mathrm{Cl}=-1.09$ to -0.45 ) and women $(\mathrm{RC}$ trend $=-0.94 ; 95 \% \mathrm{Cl}=-1.41$ to $-0.46)$ when analysed separately. The rate ratio for men versus women varied from 0.92 in 1986 to 1.26 in 1996 and 0.80 in 2006. The separate trend curves for patients aged $40-64$ years and $\geq 65$ years and for patients of low socioeconomic status and medium to high socioeconomic status also showed a

\section{Table 1. Prevalence rates of physician-diagnosed COPD by sex, age and socioeconomic status for the period 1980 to 2006.}

\section{Population subgroup}

Men

Aged 40-64 years with low socioeconomic status

Aged 40-64 years with medium to high socioeconomic status

Aged $\geq 65$ years with low socioeconomic status

Aged $\geq 65$ years with medium to high socioeconomic status

Women

Aged 40-64 years with low socioeconomic status

Aged 40-64 years with medium to high socioeconomic status

Aged $\geq 65$ years with low socioeconomic status

Aged $\geq 65$ years with medium to high socioeconomic status
Prevalence per 1000 patients per year

$76.3 \quad 42.0$

$56.4 \quad 16.3$

$311.5 \quad 177.4$

$216.7 \quad 154.3$

$26.2 \quad 38.9$

$17.1 \quad 13.2$

$88.8 \quad 135.3$

$58.7 \quad 88.5$
Prevalence difference Regression coefficient $P$-value between 1980 and $2006(\%) \quad$ trend $(95 \% \mathrm{Cl})^{\mathrm{a}}$ for trend

\begin{tabular}{llc}
$-34.3(44.9)$ & $-0.98(-1.24$ to -0.71$)$ & $<0.001$ \\
$-40.1(71.0)$ & $-1.30(-1.56$ to -1.04$)$ & $<0.001$ \\
$-134.1(43.0)$ & $-5.17(-6.23$ to -4.12$)$ & $<0.001$ \\
$-62.4(28.8)$ & $-1.55(-2.04$ to -1.05$)$ & $<0.001$ \\
\hline & & \\
$12.7(48.7)$ & $0.61(0.44$ to 0.79$)$ & $<0.001$ \\
$-3.9(23.2)$ & $-0.033(-0.20$ to 0.13$)$ & 0.680 \\
$46.5(52.4)$ & $2.55(2.02$ to 3.09$)$ & $<0.001$ \\
$29.8(50.7)$ & $2.25(1.85$ to 2.65$)$ & $<0.001$
\end{tabular}

${ }^{a}$ The regression coefficient trend reflects the annual change in prevalence per 1000 patients per year. 
statistically significant decline. After combining sex with age and socioeconomic status, the number of exacerbations became too small to estimate trend regression coefficients.

Figure 3 shows trends in patients with COPD who have exacerbations for the total COPD cohort and for men and women separately. In 1986, 20.4\% of patients with COPD reported one exacerbation and $9.2 \%$ reported two or more exacerbations. In 2006 , the percentage of patients with exacerbations had decreased to $14.6 \%$ for patients with one exacerbation $(\mathrm{RC}$ trend $=-0.26,95 \% \mathrm{Cl}=-0.42$ to -0.11 ) and to $6.4 \%$ for patients with two or more exacerbations $(\mathrm{RC}$ trend $=-0.19,95 \% \mathrm{Cl}=-0.29$ to -0.093). The separate trend curves for male and female patients with COPD who have exacerbations also showed a significant decrease. The trend curves by age and socioeconomic status were comparable to the trend curves by sex. Again, the number of patients having exacerbations became too small to conduct further analyses when combining sex with age and socioeconomic status.

\section{DISCUSSION}

\section{Summary of main findings}

Using data from the Continuous Morbidity Registration Nijmegen, this study demonstrated that the overall prevalence of physician-diagnosed COPD has decreased significantly during the last decades. The annual rates of exacerbations and proportions of patients with COPD who had exacerbations had also decreased. These findings suggest a reduction rather than an increase of the COPD burden on Dutch primary care.

When analysing prevalence trends by sex, age, and socioeconomic status a significant increase of COPD prevalence in women was observed, in particular in those aged $\geq 65$ years of low socioeconomic status. In men, on the other hand, the prevalence of COPD had decreased, independent of age and socioeconomic status. The decreasing trend curves for exacerbation rates and percentage of patients with exacerbations were independent of sex, age, and socioeconomic status.

\section{Strengths and limitations of the study}

The strengths of the current study are the long follow-up period and the standardised way of recording COPD and COPD exacerbations in all four general practices. The decision to present prevalence rates from the period 1980 and onwards was mainly based on the validity of the data prior to 1980. As three of the four practices started with morbidity registration in 1967 and the fourth practice joined the registration network in 1971, the first years after 1967 were considered as a 'run-in period'. When studying all COPD data in the Continuous Morbidity Registration, very low prevalence rates were found in the first years of registration followed by a sharp increase in the following years. This 'run-in period' has been confirmed by the oldest generation among the current GPs in the four practices. By choosing 1980 as a starting point there was greater confidence about the validity of the data; having 2006 as the end date meant it was possible to cover a very long and unique timeframe. In addition, the information in the Continuous Morbidity Registration may be considered as complete because diagnoses made by specialists are reported back to the GP and are also entered in the database. This makes the Continuous Morbidity Registration a unique database for long-term trend analyses. However, as this study had an observational design, it cannot prove causality and, as such, the results should be interpreted with care.

Although the population of the Continuous Morbidity Registration reflects the Dutch general population regarding sex and age, ${ }^{12}$ the network consists of only four practices. This may have caused the limited annual number of recorded exacerbations when analysing the different subgroups of male and female patients. Although it seems reasonable to assume that changes in smoking patterns will have attributed to the changes in prevalence and exacerbations trends, there is a lack of valid information regarding smoking status in the Continuous Morbidity Registration. This is not unique for this registration network, but is a concern to all general practice research networks that rarely use uniform definitions to document current or past smoking habits. ${ }^{19}$

Since 1996, diagnosing COPD in the Continuous Morbidity Registration practices has changed from a physician-based diagnosis to a more objective diagnosis using lung-function measurements according to COPD guidelines..$^{20,21}$ This could have provoked a change in the overall prevalence of physician-diagnosed COPD, but this was not observed in prevalence trends from 1996 onwards.

\section{Comparison with existing literature}

Accurately estimating the prevalence of COPD very much depends on the study population and the study methods. ${ }^{22}$ Therefore, the prevalence rates in the current study should be compared with other prevalence rates based on physician-reported diagnoses. Although a higher prevalence rate was found than the UK trend study published in 2000, ${ }^{6}$ the finding is in line with a recent study that showed a pooled prevalence of $5.2 \%(95 \% \mathrm{Cl}=3.3 \%$ to $7.9 \%)^{23}$ 
The gradual decline in the prevalence of COPD between 1980 and 2006 seems to be mainly caused by the sharp decrease of the prevalence in men, independent of age and socioeconomic status. The rise of COPD prevalence in women has been reported previously. ${ }^{5,6}$ However, the current study is the first to actually demonstrate that the strongest increase is in women aged $\geq 65$ years of low socioeconomic status. This finding is likely to reflect the increase of tobacco use in this subgroup during the last decades. Between 1960 and 1981, smoking rates have decreased sharply in Dutch men and recently smoking rates have also started to decrease in women, in particular those aged 20-34 years. ${ }^{25}$ If this decline continues, the increasing prevalence of COPD in women is likely to have its peak in the next decades.

It could be questioned whether the current results reflect the true burden of the disease in primary care as GPs tend to under-diagnose COPD ${ }^{26,27}$ and patients may fail to present their symptoms. ${ }^{28} \mathrm{~A}$ third of current smokers in general practice has undiagnosed COPD ${ }^{29}$ although a substantial proportion shows respiratory symptoms that need treatment. ${ }^{26}$ This implies that the true burden of COPD in primary care is far more extensive than would be expected when based on the results of the current study.

So far, there are no studies that show trends in exacerbation rates in patients in primary care. With overall annual exacerbations rates between 44.1 (1980) and 31.5 exacerbations (2006) per 100 patients with COPD, the mean exacerbation rate per patient was less than one reported exacerbation per 2 years in the current study. In addition, less than $30 \%$ of patients with COPD contributed to the total annual exacerbation rate and less than $10 \%$ could be considered as frequent exacerbaters, that is, patients with COPD who have two or more exacerbations. ${ }^{20}$ However, previous studies demonstrated that many patients have problems in recognising symptom aggravation ${ }^{30}$ and fail to report the exacerbation to their health professional. ${ }^{31}$ Although there is a relationship between age and the risk of underreporting, ${ }^{32}$ no difference was found in reported exacerbations between patients aged 40-64 years and patients aged $\geq 65$ years. Also, there was no confirmation of the relationship between low socioeconomic status and the risk for exacerbations reported from secondary-care studies. ${ }^{8-10}$

Although there is no straightforward explanation for the declining exacerbation trends, two different developments may have contributed to these findings. Vaccines and several pharmacological treatments (such as long-acting bronchodilators and inhaled corticosteroids) that may reduce exacerbation frequency and severity have been introduced in the last two decades; ${ }^{8}$ in addition, in 1992 the Dutch College of General Practitioners (NHG) introduced the first guideline on COPD management with a specific focus on the treatment of acute exacerbations. ${ }^{33}$ This may have caused a change in the attitude of Dutch GPs towards the prevention and treatment of exacerbations.

\section{Implications for future research and clinical practice}

This study demonstrated that the prevalence of physician-diagnosed COPD has decreased in recent decades. Although this study did not investigate the prevalence of undiagnosed COPD in the practice population, GPs should be aware that many patients are still undiagnosed. The finding that the increasing prevalence of COPD in women is particularly noted in those aged $\geq 65$ years with a low socioeconomic status indicates the need to focus more on this subgroup in COPD diagnosing, management, and research. The decrease in the number of recorded exacerbations and the decrease in the percentage of patients with COPD who have exacerbations are encouraging. However, as previously stated, the underreporting of worsening symptoms is a major concern in the management of patients with COPD. Improving patient understanding of the nature of an exacerbation and early recognition of its symptoms could benefit its reporting.

In conclusion, this is the first study that reports on long-term trends in COPD prevalence and exacerbation rates in primary care using unique registration data from the Continuous Morbidity Registration Nijmegen. The results from this study give more insight into trend changes in different demographic subgroups and may help GPs to redirect care and to diminish the burden of COPD.

\section{Funding body}

Erik Bischoff is recipient of a MD-clinical research trainee stipend of the Netherlands organization for health research and development (ZonMw)

\section{Ethics committee}

Not applicable

\section{Competing interests}

The authors have stated that there are none

Acknowledgements

Thanks to all the GPs of the four practices of the Continuous Morbidity Registration Nijmegen.

\section{Discuss this article}

Contribute and read comments about this article on the Discussion Forum: http://www.rcgp.org.uk/bjgp-discuss

\section{REFERENCES}

1. Wouters EF. Economic analysis of the Confronting COPD survey: an overview of results. Respir Med 2003; 97 Suppl C: S3-14.

2. Sullivan SD, Ramsey SD, Lee TA. The economic burden of COPD. Chest 2000; $117(2$ Suppl): 5S-9S. 
3. Seemungal TA, Donaldson GC, Paul EA, et al. Effect of exacerbation on quality of life in patients with chronic obstructive pulmonary disease. Am J Respir Crit Care Med 1998; 157(5 Pt 1): 1418-22.

4. Lacasse Y, Brooks D, Goldstein RS. Trends in the epidemiology of COPD in Canada, 1980 to 1995 . COPD and Rehabilitation Committee of the Canadian Thoracic Society. Chest 1999; 116(2): 306-313.

5. Mannino DM, Homa DM, Akinbami LJ, et al. Chronic obstructive pulmonary disease surveillance--United States, 1971-2000. MMWR Surveill Summ 2002; 51(6): 1-16.

6. Soriano JB, Maier WC, Egger P, et al. Recent trends in physician diagnosed COPD in women and men in the UK. Thorax 2000; 55(9): 789-794.

7. Prescott E, Vestbo J. Socioeconomic status and chronic obstructive pulmonary disease. Thorax 1999; 54(8): 737-741.

8. Wedzicha J, Seemungal T. COPD exacerbations: defining their cause and prevention. Lancet 2007; 370(9589): 786-796.

9. Tsoumakidou M, Siafakas N. Novel insights into the aetiology and pathophysiology of increased airway inflammation during COPD exacerbations. Respir Res 2006; 7: 80.

10. Hallin R, Koivisto-Hursti U, Lindberg E, Janson C. Nutritional status, dietary energy intake and the risk of exacerbations in patients with chronic obstructive pulmonary disease (COPD). Respir Med 2006; 100(3): 561-567.

11. Van Weel C, de Grauw W. Family practices registration networks contributed to primary care research. J Clin Epidemiol 2006; 59(8): 779-783.

12. Van Weel C. The Continuous Morbidity Registration Nijmegen: Background and history of a Dutch general practice database. Eur I Gen Pract 2008; 14 (Suppl 1): 5-12.

13. Research Committee of the Council of the College of General Practitioners. A classification of disease. J Coll Gen Pract 1959; 2 140-159.

14. WONCA. An international glossary for primary care. Classification Committee of WONCA in collaboration with the WHO. International Classification of Health Problems in Primary Care-2-defined. 3rd edn. London: Oxford University Press; 1983.

15. Schers $\mathrm{H}$, Bor $\mathrm{H}$, van den Hoogen $\mathrm{HJ}$, van Weel C. What went and what came? Morbidity trends in general practice from the Netherlands. Eur J Gen Pract 2008; 14 Suppl 1: 13-24.

16. Van Weel C. Validating long term morbidity recording. J Epidemiol Community Health 1995; 49 Suppl 1: 29-32.

17. Van Weel-Baumgarten EM, van den Bosch WJ, van den Hoogen HJ, Zitman FG. The validity of the diagnosis of depression in general practice: is using criteria for diagnosis as a routine the answer? $\mathrm{Br} J$ Gen Pract 2000; 50(453): 284-287.

18. Bakker B, Sieben I. Maten voor prestige, sociaaleconomische status en sociale klasse voor de standaard beroepenclassificatie 1992. Measurements for prestige, socioeconomic status and social class for the Standard Classification of Occupations 1992]. Sociale Wetenschappen 1997; 40: 1-22.

19. Wilson A, Manku-Scott T, Shepherd D, Jones B. A comparison of individual and population smoking data from a postal survey and general practice records. Br J Gen Pract 2000; 50(455): 465-468.

20. Smeele IJ, van Weel C, van Schayck CP, et al. NHG-Standaard COPD [NHG Guideline COPD]. Huisarts Wet 2007; 50(8): 362-379.

21. Rabe KF, Hurd S, Anzueto A, et al. Global strategy for the diagnosis, management, and prevention of chronic obstructive pulmonary disease: GOLD executive summary. Am J Respir Crit Care Med 2007; 176(6): 532-555.

22. Halbert RJ, Isonaka S, George D, Iqbal A. Interpreting COPD prevalence estimates: What is the true burden of disease? Chest 2003 123(5): 1684-1692.

23. Halbert RJ, Natoli JL, Gano A, et al. Global burden of COPD: systematic review and meta-analysis. Eur Respir J 2006; 28(3): 523-532.

24. Janssen-Heijnen ML, Nab HW, Van Reek J, et al. Striking changes in smoking behaviour and lung cancer incidence by histological type in south-east Netherlands, 1960-1991. Eur J Cancer 1995; 31A(6): 949-952.

25. Willemsen MC, van Leent-Loenen HM. Neemt het aantal mensen dat rookt toe of af? [Does the prevalence of smokers decrease or increase?] In: Volksgezondheid Toekomst Verkenning, Nationaal Kompas Volksgezondheid. Bilthoven: RIVM, 2007. http://www.rivm.nl/vtv/object_document/o1210n19085.html (accessed 16 Jun 2009).
26. Bednarek M, Maciejewski J, Wozniak M, et al. Prevalence, severity and underdiagnosis of COPD in the primary care setting. Thorax 2008; 63(5): 402-407.

27. Tinkelman DG, Price D, Nordyke RJ, Halbert RJ. COPD screening efforts in primary care: what is the yield? Prim Care Respir J 2007; 16(1): 49-53.

28. Van Weel C. Underdiagnosis of asthma and COPD: is the general practitioner to blame? Monaldi Arch Chest Dis 2002; 57(1): 65-68.

29. Vandevoorde J, Verbanck S, Gijssels L, et al. Early detection of COPD: A case finding study in general practice. Respir Med 2007; 101(3): 525-530.

30. Kessler R, Stahl E, Vogelmeier C, et al. Patient understanding detection, and experience of COPD exacerbations: an observational, interview-based study. Chest 2006; 130(1): 133-142.

31. Wilkinson TM, Donaldson GC, Hurst JR, et al. Early therapy improves outcomes of exacerbations of chronic obstructive pulmonary disease. Am J Respir Crit Care Med 2004; 169(12): 1298-12303.

32. Langsetmo L, Platt RW, Ernst P, Bourbeau J. Underreporting exacerbation of chronic obstructive pulmonary disease in a longitudinal cohort. Am J Respir Crit Care Med 2008; 177(4): 396-401.

33. Van der Waart MA, Dekker FW, Nijhoff S, et al. NHG-standaard CARA bij volwassenen: behandeling [NHG Guideline on Chronic Non-specific Lung Diseases in adults: treatment]. Huisarts Wet 1992; 35(11): 437-443. 